\title{
Instabilities of Nanoflow Displacements in Homogeneous Porous Media
}

\author{
Behnam Dastvareh, Jalel Azaiez \\ Department of Chemical and Petroleum Engineering, Schulich School of Engineering, University of Calgary \\ Calgary, Alberta, Canada, T2N 1N4 \\ behnam.dastvareh@ucalgary.ca; azaiez@ucalgary.ca
}

\section{Extended Abstract}

The instabilities at the interface of the two flowing fluids in porous media will grow as finger-shape patterns if the viscosity of the displacing fluid is lower than that of the displaced one [1]. This phenomenon called viscous fingering (VF) is observed in different applications such as in-situ oil recovery, chromatographic columns and contaminant transport in soil. Viscous fingering can be either a favorable or an unfavorable phenomenon. It is unfavorable if the highest sweep of the displaced fluid is desired, while it is favorable where the enhancement of the mixing rate of the fluids is desired in very low Reynolds number flows [2].

Due to the advantageous features of nanoparticles, nanofluids, the suspension of nanoparticles in a base fluid, are widely used in different applications. Regrading to their transport in porous media which occurs in applications such as oil recovery and ground water remediation, the effect of VF should not be ignored. However, despite the importance of the issue, there are lacks of studies on this topic. The objective of this study is to investigate the effects of the presence of nanoparticles on the instability criteria and finger configuration in miscible flow displacements. The results will be useful and helpful in controlling the growing instabilities in related applications.

The governing equations include the conservation of mass, conservation of momentum in the form of Darcy's law and three convection-diffusion equations representing the transport of the fluids and nanoparticles. The equations are solved numerically using the Hartley-based pseudo-spectral method. Using this method, the equations are transferred to the Hartley space where the special derivatives are eliminated from the equations and accordingly the errors encountered with the numerical derivatives will be reduced. Furthermore, the governing equations are changed to a system of ODEs and algebraic equations in the Hartley space, and as a result are easier to solve with a high degree of accuracy [3].

The intensity of the instability can be observed qualitatively by the shape and number of fingers. However, the analysis of the mixing length, the ratio of the length within a specific concentration range to the length of the whole domain, breakthrough time, the time for fingers to reach the end of the domain, and contact line length, the length of the iso-surface of a specific concentration will help to quantify the degree of instability. The results show that the presence of nanoparticles in the displacing fluid attenuates the instability of an initially unstable binary system. However, the degree of the deposition of the nanoparticles seems to affect the intensity of this instability. The finger configuration and further quantitative analysis are conducted in terms of the nanofluid viscosity and nanoparticle deposition rate for different flow scenarios, and the results will be discussed and interpreted.

\section{References}

[1] G. M. Homsy, "Viscous fingering in porous media," Annual review of fluid mechanics, vol. 19, pp. 271$311,1987$.

[2] B. Jha, L. Cueto-Felgueroso, and R. Juanes, "Fluid Mixing from Viscous Fingering," Physical Review Letters, vol. 106, p. 194502, 2011.

[3] M. Islam and J. Azaiez, "Fully implicit finite difference pseudo-spectral method for simulating high mobility-ratio miscible displacements," International journal for numerical methods in fluids, vol. 47, pp. 161-183, 2005. 